\title{
Canonical Angular Momentum of Electron, Positron and the Gamma Photon
}

\author{
Ziya Saglam1, Mesude Saglam² \\ ${ }^{1}$ Department of Physics, Aksaray University, Aksaray, Turkey \\ ${ }^{2}$ Department of Physics, Ankara University, Ankara, Turkey \\ Email: zsaglam@aksaray.edu.tr, saglam@science.ankara.edu.tr
}

Received 17 November 2015; accepted 20 January 2016; published 25 January 2016

Copyright (C) 2016 by authors and Scientific Research Publishing Inc.

This work is licensed under the Creative Commons Attribution International License (CC BY).

http://creativecommons.org/licenses/by/4.0/

\section{(c) (i) Open Access}

\section{Abstract}

We calculate the canonical angular momentum of a free electron, positron and gamma photon. We show that for any particle with charge $q$ the canonical angular momentum $\left(J_{c}\right)$ is written as the summation of the kinetic angular momentum $\left(J_{k i n}\right)$ and the intrinsic quantum flux $\left(\Phi_{i n t}\right)$ dependent terms. In terms of the z-components this can be written as $\left(J_{c}\right)_{z}=\left(J_{k i n}\right)_{z}+\left(\frac{|q|}{2 \pi}\right) \phi_{\text {int }}$. For a free electron $\left(e^{-}\right)$and a positron $\left(e^{+}\right)$depending on the spin orientation we find that: $\left(J_{c}\right)_{z}=\left(e^{-\uparrow}\right)_{z}=0 ;\left(J_{c}\right)_{z}=\left(e^{-} \downarrow\right)_{z}=0 ;\left(J_{c}\right)_{z}=\left(e^{+} \uparrow\right)_{z}=\hbar$ and $\left(J_{c}\right)_{z}=\left(e^{+} \downarrow\right)_{z}=-\hbar$ respectively. Similarly for a gamma $(\gamma)$ photon, propagating in $\mathrm{z}$ direction with an angular frequency $\omega$, the canonical angular momentum is found to be: $\left(J_{c}(\gamma)\right)_{z}= \pm \hbar$, here the $(+)$ and $(-)$ signs stand for the right and left hand circular helicity respectively.

\section{Keywords}

Magnetic Moment, Quantum Flux, Gamma Photons, Canonical Angular Momentum, Electron-Positron Annihilation, Right (Left) Hand Circular Helicity

\section{Introduction}

Conservation of physical quantities such as energy, linear and angular momenta in classical and quantum collisions is an important tool to find the other physical quantities of the collision systems. But the conservation of the canonical angular momentum has not been studied in detail. In a recent study Saglam and Sahin [1] calcu- 
lated the intrinsic quantum flux of gamma photons by using the conservation of the canonical angular momentum and showed that depending on its helicity, a gamma photon carried an intrinsic quantum flux of $\pm \phi_{0}= \pm h / e$ along the propagation direction. Here the $(+)$ and $(-)$ signs stand for the right hand $(r h)$ and left hand $(I h)$ circular helicity respectively. In the present study we calculate the spin dependent canonical angular momenta of a free electron, positron and gamma photons. We first show that for any particle with the charge $q$ the canonical angular momentum $\left(J_{c}\right)$ has two parts: the kinetic angular momentum term $\left(\boldsymbol{J}_{k i n}\right)$ and the intrinsic flux $\left(\phi_{\text {int }}\right)$ dependent terms. In terms of the z-components we write: $\left(\boldsymbol{J}_{c}\right)_{z}=\left(\boldsymbol{J}_{\text {kin }}\right)+(|q| / 2 \pi) \phi_{\text {int }}$. The difference between $\boldsymbol{J}_{c}$ and $\boldsymbol{J}_{k i n}$ is due to the presence of the intrinsic magnetic field and hence the intrinsic quantum flux, $\phi_{\text {int }}$, which has already been calculated [1] [2]. For a free electron $\left(e^{-}\right)$and a positron $\left(e^{+}\right)$, depending on the spin orientations, spin-up $(\uparrow)$ or spin-down $(\downarrow)$, we have: $\phi_{\text {int }}\left(e^{-} \uparrow\right)=-\phi_{0} / 2$; $\phi_{\text {int }}\left(e^{-} \downarrow\right)=\phi_{0} / 2 ; \quad \phi_{\text {int }}\left(e^{+} \uparrow\right)=-\phi_{0} / 2 ; \quad \phi_{\text {int }}\left(e^{+} \downarrow\right)=-\phi_{0} / 2$ respectively. Similarly for a gamma photon, propagating in z direction with an angular frequency $\omega$, we have $\phi_{\text {int }}(\gamma)= \pm \phi_{0}$ along the propagation direction. Here the $(+)$ and $(-)$ signs stand for the $(r h)$ and $(I h)$ circular helicity respectively. The canonical angular momentum is always represented by $j_{c}=-i \hbar \partial / \partial \varphi$ and its eigenvalues are $m \hbar(m \in Z)$. Therefore the spectrum of kinetic angular momentum operator $\left(J_{k i n}\right)$ consists of $\hbar$ times an integer number $m$ shifted by $|q| \phi_{\text {int }} / 2 \pi$. In the present study the sources of these intrinsic flux and hence the magnetic fields are due to the magnetic moments of these particles.

When we do the Stern-Gerlach experiment (SGE) with these particles the magnetic field gradient in the SternGerlach device serves as a detector for the particle's magnetic moment vector: If the non-uniformity of the magnetic field is along the $\mathrm{z}$ direction such as $\boldsymbol{B}=B_{z}(\mathrm{z}) \hat{z}$, then because of the torque $\boldsymbol{\tau}=\boldsymbol{\mu} \times \boldsymbol{B}$ on these particles, the magnetic moment vector can have z-component only: $\boldsymbol{\mu}=\mu_{z} \hat{z}$ (where $\mu_{z} \geq 0$ or $\mu_{z} \leq 0$ ). On the other hand the potential energy $U=-\boldsymbol{\mu} \cdot \boldsymbol{B}$ produces a force of $\boldsymbol{F}=\boldsymbol{\mu}_{z}\left(\frac{\partial B_{z}}{\partial z}\right) \hat{z}$ which has quantized values as $\mu_{z}$. Depending on whether $\mu_{z} \geq 0$ or $\mu_{z} \leq 0$, the particles are deflected upward or downward. For example, in the SGE with electron, the electron

beam is deflected into two sub-beams which mean that $\mu_{\mathrm{z}}$ takes only two possible quantized values which are equal to $\mu_{z}= \pm \mu_{B}= \pm\left(e \hbar / 2 m_{0}\right)$ where $\mu_{B}$ is the Bohr magneton. Because of the negative charge of the electron, the relation between spin and the magnetic moment was set as $\boldsymbol{\mu}=-g \mu_{\mathrm{B}} \boldsymbol{S}$ where $g$ is the Lande-g factor which is equal to 2 for a free electron and $\hbar \boldsymbol{S}$ is the spin angular momentum vector. So in the SGE with electron, the z component of spin $\left(S_{z}\right)$ takes only two possible values which are: $S_{z} \equiv m_{s}= \pm 1 / 2$. Therefore if $\left(\partial B_{z} / \partial z\right)>0$ then the spin-down electrons will be deflected upward while the spin-up ones will be deflected downward. Similarly if we have SGE with a positron beam, because of the positive charge, the spin-down positrons will be deflected downward while the spin-up ones will be deflected upward. But when it comes to the gamma photons, which are the composite particles made up of an electron and a positron, the direction of the deflection is determined by the sign of the $\Sigma \mu_{\mathrm{z}}$. Therefore the magnetic moment of the gamma photon is an important property (not spin) to determine the direction of the deflection. Recently we have calculated [3] the magnetic moment of a gamma photon and found that the z-component of the magnetic moment is equal to $\mu_{z}= \pm\left(e c^{2} / \omega\right)$ where $\omega$ is the angular frequency of photon. Here the $\left(^{+}\right)$and $(-)$signs correspond to (rh) and $(I h)$ circular helicity respectively.

\section{Canonical Angular Momentum of a Free Electron and a Positron}

The definition of the canonical angular momentum vector, $\boldsymbol{J}_{c}$, for a free particle with charge $q$ and the position vector $\boldsymbol{r}$ is given by [1]:

$$
\boldsymbol{J}_{c}=\boldsymbol{r} \times \boldsymbol{p}_{\text {tot }}=\boldsymbol{r} \times\left(\boldsymbol{p}_{\text {kin }}+\boldsymbol{p}_{\text {field }}\right)=\boldsymbol{r} \times\left(\boldsymbol{p}_{\text {kin }}+q \boldsymbol{A}\right)
$$

which is put in the form:

$$
\left(\boldsymbol{J}_{c}\right)_{z}=\left(\boldsymbol{J}_{\text {kin }}\right)_{z}+(|q| / 2 \pi) \phi_{\text {int }}
$$

where $\boldsymbol{J}_{k i n}$ is the kinetic angular momentum and $\phi_{i n t}$ is the intrinsic quantum flux of the particle.

For a free electron ( $\left.\mathrm{e}^{-}\right)$and a positron $\left(\mathrm{e}^{+}\right)$we will have only the spin contribution to the kinetic angular mo- 
mentum. Therefore for spin-up $(\uparrow)$ and spin-down $(\downarrow)$ directions we write:

$$
\begin{aligned}
& \left(\boldsymbol{J}_{\text {kin }}\left(e^{-\uparrow}\right)\right)_{z} \equiv \hbar\left(\boldsymbol{S}\left(e^{-} \uparrow\right)\right)_{z}=\hbar / 2 ;\left(\boldsymbol{J}_{k i n}\left(e^{-} \downarrow\right)\right)_{z} \equiv \hbar\left(\boldsymbol{S}\left(e^{-} \downarrow\right)\right)_{z}=-\hbar / 2 \\
& \left(\boldsymbol{J}_{k i n}\left(e^{+} \uparrow\right)\right)_{z} \equiv \hbar\left(\boldsymbol{S}\left(e^{+} \uparrow\right)\right)_{z}=\hbar / 2 ;\left(\boldsymbol{J}_{k i n}\left(e^{+} \downarrow\right)\right)_{z} \equiv \hbar\left(\boldsymbol{S}\left(e^{+} \downarrow\right)\right)_{z}=-\hbar / 2
\end{aligned}
$$

respectively. Here $\hbar \boldsymbol{S}$ is the spin angular momentum vector. For a free electron $\left(e^{-}\right)$and a positron $\left(e^{+}\right)$ corresponding intrinsic flux for spin-up $(\uparrow)$ and spin-down $(\downarrow)$ directions are given by [1]:

$$
\begin{aligned}
& \Phi_{\text {int }}\left(e^{-\uparrow}\right)=-\frac{h}{2 e}=-\frac{\Phi_{0}}{2} ; \quad \Phi_{\text {int }}\left(e^{-} \downarrow\right)=\frac{h}{2 e}=\frac{\Phi_{0}}{2} \\
& \Phi_{\text {int }}\left(e^{+} \uparrow\right)=\frac{h}{2 e}=\frac{\Phi_{0}}{2} ; \quad \Phi_{\text {int }}\left(e^{+} \downarrow\right)=-\frac{h}{2 e}=-\frac{\Phi_{0}}{2}
\end{aligned}
$$

Substituting $\left|q\left(e^{-}\right)\right|=\left|q\left(e^{+}\right)\right|=e$ and (3a)-(4b) in (2) we find the z-components of the canonical angular momenta of a free electron $\left(\mathrm{e}^{-}\right)$and a free positron $\left(\mathrm{e}^{+}\right)$for spin-up $(\uparrow)$ and spin-down $(\downarrow)$ directions:

$$
\begin{array}{ll}
\left(J_{c}\left(e^{-} \uparrow\right)\right)_{z}=0 ; & \left(J_{c}\left(e^{-} \downarrow\right)\right)_{z}=0 \\
\left(J_{c}\left(e^{+} \uparrow\right)\right)_{z}=\hbar ; & \left(J_{c}\left(e^{+} \downarrow\right)\right)_{z}=-\hbar
\end{array}
$$

respectively.

\section{Canonical Angular Momenta of Gamma Photons}

To calculate the canonical angular momentum of a gamma photon $(\gamma)$ our starting point will be the electron-positron annihilation process ending with the creation of two gamma photons with $(r h)$ and $(I h)$ circular helicities [1]. We will assume that electron and positron collide at the origin of the coordinate system. After the collision we will have two photons with the same energy:

$$
E=m_{0} c^{2}=\hbar \omega=(\hbar k c=h c / \lambda)
$$

but with different helicities $(r h)$ and $(I h)$ circular helicities. Here $m_{0} c^{2}$ is the rest mass energy of both $\left(e^{-}\right)$and $\left(e^{+}\right)$and $\eta \omega$ is the energy of each gamma photon created with the same angular frequency $\omega$. Following Saglam and Sahin [1], we use Dirac notations to represent the wave functions of two gamma photons with $(r h)$ and $(l h)$ circular helicities:

$$
\varnothing(r h)=\left|e^{-} \downarrow\right\rangle\left|e^{+} \uparrow\right\rangle ; \quad \varnothing(l h)=\left|e^{-\uparrow}\right\rangle\left|e^{+} \downarrow\right\rangle
$$

For a gamma photon propagating in $\mathrm{z}$ direction with the wave vector momentum relation given in (2) becomes:

$$
\left(\boldsymbol{J}_{c}\right)_{z}=\hbar(\boldsymbol{S})_{z}+(|q| / 2 \pi) \phi_{i n t}
$$

Next we calculate the expectation values of spin and the the intrinsic flux for gamma photons with $(r h)$ and (lh) circular helicities: In Appendix, we show that for both helicities the expectation values of $(\boldsymbol{S})_{z}$ are zero. We also show that for a gamma $(\gamma)$ photon, propagating in z direction, we have $\Phi_{\text {int }}(\gamma)= \pm \Phi_{0}$ along the propagation direction. Here the $(+)$ and $(-)$ signs stand for with $(r h)$ and $(I h)$ circular helicity respectively. Now using the current-loop model [1] [2] for gamma photons, we can assume that the above intrinsic flux $\Phi_{\text {int }}(\gamma)$ are caused by the fictitious point charge $\left(q_{f i c}=e\right)$ rotating in the helical sense with $(r h)$ or $(I h)$ with the angular frequency $\omega$ and moving in z direction with the velocity of light. Further in the current loop model it is also proved that the z-component of the magnetic field is uniform throughout the loop [4]. Substituting $\boldsymbol{S}_{z}(r h)=\boldsymbol{S}_{z}(I h)=0, \phi_{\text {int }}(r h)=\phi_{0}, \quad \phi_{\text {int }}(I h)=-\phi_{0}$ and $|q|=e$ in (8) we find the canonical angular mo- 
menta of gamma $(\gamma)$ photons with $(r h)$ and $(I h)$ circular helicities:

$$
\left(\boldsymbol{J}_{c}(\gamma(r h))\right)_{z}=\hbar ; \quad\left(\boldsymbol{J}_{c}(\gamma(I h))\right)_{z}=-\hbar
$$

\section{Conclusion}

We have calculated the canonical angular momentum of a free electron, positron and gamma photon. We show that for any particle with charge $q$ the canonical angular momentum $\left(J_{c}\right)$ is written as the summation of the kinetic angular momentum $\left(J_{k i n}\right)$ and the intrinsic quantum flux $\left(\phi_{\text {int }}\right)$ dependent terms. In terms of the zcomponents, we show that: $\left(\boldsymbol{J}_{c}\right)_{z}=\left(\boldsymbol{J}_{\text {kin }}\right)_{z}+(|q| / 2 \pi) \phi_{\text {int }}$. For a free electron $\left(e^{-}\right)$and a positron $\left(e^{+}\right)$, depending on the spin orientation, we find that: $\left(\boldsymbol{J}_{c}\left(e^{-\uparrow}\right)\right)_{z}=0 ;\left(\boldsymbol{J}_{c}\left(e^{-} \downarrow\right)\right)_{z}=0 ;\left(\boldsymbol{J}_{c}\left(e^{+} \uparrow\right)\right)_{z}=\hbar$ and $\left(\boldsymbol{J}_{c}\left(e^{+} \uparrow\right)\right)_{z}=-\hbar$ respectively. Similarly for a gamma $(\gamma)$ photon, propagating in z direction with an angular frequency $\omega$, the canonical angular momentum is found to be: $\left(\boldsymbol{J}_{c}(\gamma)\right)_{z}= \pm \hbar$. Here the $\left(^{+}\right)$and $(-)$signs stand for the right and left hand circular helicity respectively. The present result will help for a better understanding of the photonic transitions in atoms, nano-structures and so on. A more detailed study will be presented in the future.

\section{References}

[1] Saglam, M. and Sahin, G. (2009) International Journal of Modern Physics B, 23, 4977-4985. http://dx.doi.org/10.1142/S0217979209053862

[2] Saglam, M. and Boyacioglu, B. (2002) International Journal of Modern Physics B, 16, 607-614. http://dx.doi.org/10.1142/s0217979202010038

[3] Saglam, Z. and Sahin, G. (2015) Journal of Modern Physics, 6, 937-947. http://dx.doi.org/10.4236/jmp.2015.67098

[4] Saglam, M. (2002) Is Electron an Anyon with Spin 1/2? http://www.arxiv.org/abs/physics/0205038 
Appendix: Calculation of the Expectation Values of $\sum S_{2}$ and $\sum \phi_{i n t}$ for Gamma Photons

To calculate of the expectation values of of $\sum S_{2}$ and $\sum \phi_{\text {int }}$ for gamma photons our starting point will be Dirac representation given in (7) of the wave functions of two gamma photons with $(r h)$ and $(I h)$ circular helicities: $\varnothing(r h)=\left|e^{-} \downarrow\right\rangle\left|e^{+} \uparrow\right\rangle ; \varnothing(l h)=\left|e^{-} \uparrow\right\rangle\left|e^{+} \downarrow\right\rangle$. We first write the normalization conditions as follows:

$$
\begin{gathered}
\iint \varnothing^{*}(r h) \varnothing(r h) \mathrm{d} V_{1} \mathrm{~d} V_{2}=\left\langle e^{-} \downarrow \| e^{-} \downarrow\right\rangle\left\langle e^{+} \uparrow \| e^{+} \uparrow\right\rangle=1 \\
\iint \varnothing^{*}(I h) \varnothing(I h) \mathrm{d} V_{1} \mathrm{~d} V_{2}=\left\langle e^{-\uparrow} \| e^{-} \uparrow\right\rangle\left\langle e^{+} \downarrow \| e^{+} \downarrow\right\rangle=1 \\
\iint \varnothing^{*}(r h) \varnothing(I h) \mathrm{d} V_{1} \mathrm{~d} V_{2}=\left\langle e^{+} \uparrow \| e^{+} \downarrow\right\rangle\left\langle e^{-} \downarrow \| e^{-\uparrow}\right\rangle=0 \\
\iint \varnothing^{*}(I h) \varnothing(r h) \mathrm{d} V_{1} \mathrm{~d} V_{2}=\left\langle e^{+} \downarrow \| e^{+} \uparrow\right\rangle\left\langle e^{-\uparrow} \| e^{-} \downarrow\right\rangle=0
\end{gathered}
$$

where $d V_{1}$ and $d V_{2}$ are the volume elements for electron and positron respectively.

The expectation values of the total z-components of the spin $\sum S_{z}=\left[\left(S_{1}\right)_{z}+\left(S_{2}\right)_{z}\right]$ for the eigenstates $\varphi(r h)$ and $\varphi(l h)$ are:

$$
\begin{aligned}
S_{z}(r h) & =\iint \varnothing^{*}(r h) \sum S_{z} \varnothing(r h) \mathrm{d} V_{1} \mathrm{~d} V_{2}=\iint \varnothing^{*}(r h)\left[\left(S_{1}\right)_{z}+\left(S_{2}\right)_{z}\right] \varnothing(r h) \mathrm{d} V_{1} \mathrm{~d} V_{2} \\
& =\left\langle e^{+} \uparrow\left|\left\langle e^{-} \downarrow\left|\left[\left(S_{1}\right)_{z}+\left(S_{2}\right)_{z}\right]\right| e^{-} \downarrow\right\rangle\right| e^{+} \uparrow\right\rangle \\
& =\left\langle e^{-} \downarrow\left|\left(S_{1}\right)_{z}\right| e^{-} \downarrow\right\rangle\left\langle e^{+} \uparrow|| e^{+} \uparrow\right\rangle+\left\langle e^{-} \downarrow \| e^{-} \downarrow\right\rangle\left\langle e^{+} \uparrow\left|\left(S_{1}\right)_{z}\right| e^{+} \uparrow\right\rangle=-\frac{1}{2}+\frac{1}{2}=0
\end{aligned}
$$

and

$$
\begin{aligned}
S_{z}(l h)_{z} & =\iint \varnothing^{*}(I h) \sum S_{z} \varnothing(I h) \mathrm{d} V_{1} \mathrm{~d} V_{2}=\iint \varnothing^{*}(l h)\left[\left(S_{1}\right)_{z}+\left(S_{2}\right)_{z}\right] \varnothing(I h) \mathrm{d} V_{1} \mathrm{~d} V_{2} \\
& =\left\langle e^{+} \downarrow \| e^{-\uparrow}\right\rangle\left[\left(S_{1}\right)_{z}+\left(S_{2}\right)_{z}\right]\left|e^{-\uparrow}\right\rangle\left|e^{+} \downarrow\right\rangle \\
& =\left\langle e^{-} \uparrow\left|\left(S_{1}\right)_{z}\right| e^{-\uparrow}\right\rangle\left\langle e^{+} \uparrow \| e^{+} \downarrow\right\rangle+\left\langle e^{-\uparrow} \| e^{-\uparrow}\right\rangle\left\langle e^{-} \downarrow\left|\left(S_{1}\right)_{z}\right| e^{-} \downarrow\right\rangle=\frac{1}{2}-\frac{1}{2}=0 .
\end{aligned}
$$

With a similar treatment we calculate the expectation values of the total intrinsic flux $\sum \phi_{\text {int }}=\left[\left(\phi_{1}\right)_{\text {int }}+\left(\phi_{2}\right)_{\text {int }}\right]$ for the eigenstates $\varphi(r h)$ and $\varphi(l h)$ are:

$$
\begin{aligned}
\Phi_{\text {int }}(r h) & =\iint \varnothing^{*}(r h) \sum \Phi_{\text {int }} \varnothing(r h) \mathrm{d} V_{1} \mathrm{~d} V_{2}=\iint \varnothing^{*}(r h)\left[\left(\phi_{1}\right)_{\text {int }}+\left(\Phi_{2}\right)_{\text {int }}\right] \varnothing(r h) \mathrm{d} V_{1} \mathrm{~d} V_{2} \\
& =\left\langle e^{+} \uparrow\left|\left\langle e^{-} \downarrow\left|\left[\left(\phi_{1}\right)_{\text {int }}+\left(\phi_{2}\right)_{\text {int }}\right]\right| e^{-} \downarrow\right\rangle\right| e^{+} \uparrow\right\rangle \\
& =\left\langle e^{-} \downarrow\left|\left(\Phi_{1}\right)_{\text {int }}\right| e^{-} \downarrow\right\rangle\left\langle e^{+} \uparrow|| e^{+} \uparrow\right\rangle+\left\langle e^{-} \downarrow \| e^{-} \downarrow\right\rangle\left\langle e^{+} \uparrow\left|\left(\phi_{2}\right)_{\text {int }}\right| e^{+} \uparrow\right\rangle=\frac{(\phi)_{0}}{2}-\frac{(\phi)_{0}}{2}=(\phi)_{0}
\end{aligned}
$$

and

$$
\begin{aligned}
& \phi_{\text {int }}(l h)=\iint \varnothing^{*}(l h) \sum \phi_{\text {int }} \varnothing(l h) \mathrm{d} V_{1} \mathrm{~d} V_{2}=\iint \varnothing^{*}(l h)\left[\left(\phi_{1}\right)_{\text {int }}+\left(\phi_{2}\right)_{\text {int }}\right] \varnothing(l h) \mathrm{d} V_{1} \mathrm{~d} V_{2} \\
& =\left\langle e^{+} \downarrow\left|\left\langle e^{-\uparrow \mid}\left|\left[\left(\phi_{1}\right)_{\text {int }}+\left(\phi_{2}\right)_{\text {int }}\right]\right| e^{-\uparrow}\right\rangle\right| e^{+} \downarrow\right\rangle \\
& =\left\langle e^{-} \uparrow\left|\left(\phi_{1}\right)_{\text {int }}\right| e^{-} \uparrow\right\rangle\left\langle e^{+} \uparrow|| e^{+} \downarrow\right\rangle+\left\langle e^{-\uparrow} \| e^{-\uparrow}\right\rangle\left\langle e^{+} \downarrow\left|\left(\Phi_{2}\right)_{\text {int }}\right| e^{+} \downarrow\right\rangle=-\frac{(\phi)_{0}}{2}+\frac{(\phi)_{0}}{2}=-(\phi)_{0}
\end{aligned}
$$

